\title{
Glueball mass spectra and other issues for supergravity duals of QCD models
}

\author{
Joseph A. Minahan \\ California Institute of Technology, \\ Pasadena, CA 91125, USA \\ E-mail: minahan@ias.edu
}

Abstract: We derive WKB expressions for glueball masses of various finite temperature supergravity models. The results are very close to recent numerical computations. We argue that the spectra has some universality that depends only on the dimension of the $A d S$ space and the singularity structure of the horizon. This explains the stability of the $0^{++}$glueball mass ratios between various models. We also consider the recently proposed nonsupersymmetric model arising from the type 0 string. In the supergravity limit of this model, the heavy quark potential has an effective coupling with $1 /(\log u)$ behavior in the $U V$. Unfortunately, the supergravity solution implies that the heavy quark potential is still coulombic in the infrared, with an effective coupling of order 1 . We also argue that the type 0 supergravity background solution does not have normalizable glueball solutions.

Keywords: Nonperturbative Effects, 17N Expansion, Brane Dynamics in Gauge Theoriesi. 


\section{Contents}

i.: Introduction

2. WKB Masses for $0^{++}$Glueballs

3. Glueball masses for generalized supergravity backgrounds

i. The type 0 nonsupersymmetric model.

5. Conclusions

\section{Introduction}

One of the many interesting developments to arise out of Maldacena's conjecture 2.2.

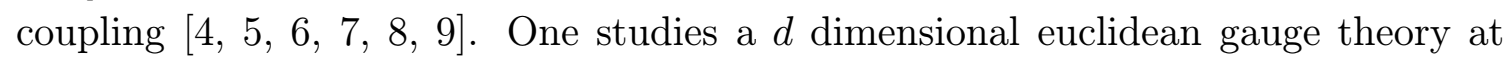
finite temperature, which is equivalent to a theory with $d-1$ noncompact directions and a Euclidean time compactified on a circle of circumference $\beta$. As was pointed out by Witten [i, the Maldacena conjecture relates wave equations in an $A d S$ blackhole background to two point correlation functions of a finite temperature Yang-Mills gauge theory.

Using this conjecture Witten argued that the dilaton wave equation in this background implies a discrete glueball spectrum with a finite gap. This spectrum was

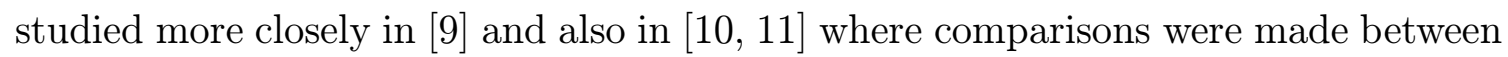
the supergravity results and lattice gauge theory results. However, the strong coupling behavior of QCD is highly nonuniversal, so there really is no reason to expect much similarity between the lattice results and the supergravity results, beyond the fact that the spectra for both theories is discrete with a finite gap.

However, one might hope to find some universality within different supergravity models. In particular, other supergravity models were recently studied that correspond to finite temperature QCD with its $R$ symmetry group broken [1] has the nice feature of getting rid of some of the unwanted Kaluza-Klein states. It was noted in [i] 3 in that there seemed to be some universality in the mass ratios of $J^{P C}=0^{++}$glueball states for the different supergravity models. One of the purposes of this paper is to explain this universality by finding WKB approximations for the glueball spectra. As it turns out, the leading order term depends on the particular 
supergravity theory being considered. However, the subleading term has universal behavior, depending only on the dimension of the $A d S$ space and the horizon singularity. One could then speculate that if "real" QCD has a supergravity dual that is asymptotically $A d S_{n}$, then it too will have some universal behavior, that depends only on $n$ and the singularity structure at a horizon.

In all of the above models, supersymmetry was broken by turning on a temperature. Therefore, supersymmetry is restored in the ultraviolet and hence the supergravity solutions do not exhibit asymptotic freedom. Recently, a nonsupersymmetric gauge model was proposed that arises from D3 branes in the nonsupersymmetric type 0 theory [i] $\left.{ }_{1}^{1} \bar{i}_{1}\right]$. Since supersymmetry is never restored, one should expect to see running of the coupling in the $U V$. In principle, one could also apply the WKB analyis to this model and derive its glueball spectrum, up to an overall scale.

The type 0 theory has a tachyon in the bulk that presumably gets an expectation

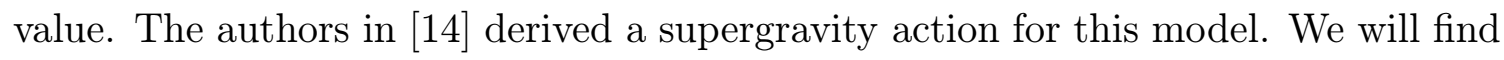
asymptotic solutions to the equations of motion coming from this supergravity action. The results found here should be taken with a grain of salt since the background metric has curvature that is either greater than or roughly equal to the string scale in both the $U V$ and $I R$. Nonetheless, one still hopes that the supergravity results are qualitatively correct, as in the case for the entropy of $\mathcal{N}=4$ super Yang-Mills at finite temperature, where the supergravity result differed from the perturbative

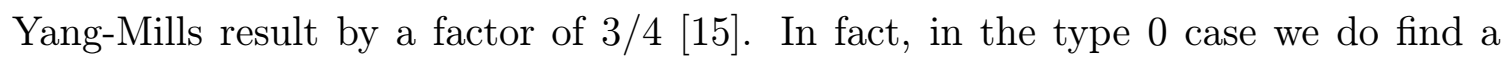
running of the effective coupling in the $U V$. However, in the infrared we do not find confinement nor do we find normalizable glueball solutions. This suggests that one must consider the full $\sigma$-model to see such behavior.

In section ${ }_{2}^{2}$ we derive WKB expressions for the masses of $0^{++}$in the finite temperature models described in [- [- We compare these results to the recent numerical results and we find excellent agreement. We also describe the six dimensional model with five uncompactified directions and argue that the glueball spectrum has a finite gap with a continuous spectrum above the gap.

In section 3 is derive WKB expressions for more general supergravity models, and we show that these expressions have a general form that depends on the dimension of the asymptotic $A d S$ space and the singularity at the horizon. This explains the recently noted stability of the spectrum for the class of models discussed in [i] Using this analysis we also find WKB expressions for the $0^{--}$and $0^{-+}$glueballs in 3 and 4 dimensions respectively. We again find good agreement with the numerical results.

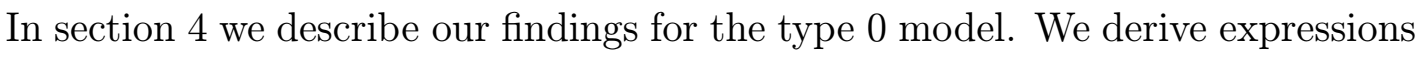
for the metric and the coupling in the ultraviolet and infrared and use this to find the heavy quark potential in these two limits. We also argue that the $I R$ behavior of the metric and coupling does not allow for normalizable glueball solutions.

In section 


\section{WKB Masses for $0^{++}$Glueballs}

In this section we compute masses using a WKB approximation for the $0^{++}$glueballs in the supergravity models in [ind. In the next section we will consider more general cases as well as the WKB solutions for the other glueballs recently discussed in the literature.

The $0^{++}$glueball spectrum is governed by the dilaton wave equation in the appropriate background. Consider first the background arising fron $N$ stationary $\mathrm{D} p$ branes at finite temperature $T$. The metric in the near horizon limit is

$$
\begin{array}{r}
d s^{2}=\alpha^{\prime}\left[\frac{U^{(7-p) / 2}}{\sqrt{g N}}\left(\left(1-\left(\frac{U_{T}}{U}\right)^{7-p}\right) d t^{2}+d x_{i}^{2}\right)+\right. \\
\left.+\frac{\sqrt{g N}}{U^{(7-p) / 2}}\left(\frac{d U^{2}}{1-\left(\frac{U_{T}}{U}\right)^{7-p}}+U^{2} d \Omega_{8-p}^{2}\right)\right]
\end{array}
$$

and with a dilaton background

$$
e^{\phi}=g\left(\frac{U^{7-p}}{g N}\right)^{(p-3) / 4}
$$

The temperature $T$ is related to $U_{T}$ and the coupling by

$$
T=\frac{7-p}{4 \pi} \frac{U_{T}^{(5-p) / 2}}{\sqrt{g N}} .
$$

The dilaton equation of motion is

$$
\partial_{\mu} e^{-2 \phi} \sqrt{g} g^{\mu \nu} \partial_{\nu} \phi=0
$$

Assuming that $\phi$ is of the form $\phi=e^{i k \cdot x} \rho(u)$ with $k^{2}=-M^{2}$, (4.2.

$$
\partial_{U}\left(U^{7-p}-U_{T}^{7-p}\right) U \partial_{U} \phi+M^{2} g N U \phi=0 .
$$

Defining a new variable $x=U^{2} / U_{T}^{2}$ and rescaling, (2.5.5.5

$$
\partial\left(x^{2+1 / n}-x\right) \partial \phi+\lambda \phi=0
$$

where $n=2 /(5-p)$ and

$$
\lambda=M^{2}\left(\frac{n+1}{4 \pi n T}\right)^{2} .
$$

The differential equation in ('드. roots of unity, so solutions to this equation are unknown for finite $n$. In order to do the WKB approximation, we define a new function $\psi=\sqrt{\frac{x-1}{x^{2+1 / n}-x}} \phi$, and we change variables to $x=1+e^{y}$. The equation in $\left(2_{2}^{2} \overline{6}_{1}^{1}\right)$ now takes the form

$$
\psi^{\prime \prime}+\left(\frac{\lambda}{f} e^{y}-\frac{1}{2} \frac{f^{\prime \prime}}{f}+\frac{1}{4}\left(\frac{f^{\prime}}{f}\right)^{2}\right) \psi=0,
$$


where the primes denote derivatives with respect to $y$ and $f=\left(1+e^{-y}\right)\left(\left(e^{y}+1\right)^{1+1 / n}-\right.$ 1).

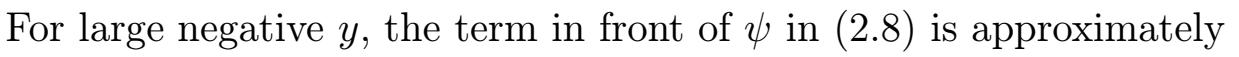

$$
\frac{\lambda}{f} e^{y}-\frac{1}{2} \frac{f^{\prime \prime}}{f}+\frac{1}{4}\left(\frac{f^{\prime}}{f}\right)^{2} \approx\left(\frac{\lambda n}{1+n}-\frac{1}{4}(2+1 / n)\right) e^{y} \quad y \ll 0 .
$$

For large positive $y$ the asymptotic behavior for this term is

$$
\frac{\lambda}{f} e^{y}-\frac{1}{2} \frac{f^{\prime \prime}}{f}+\frac{1}{4}\left(\frac{f^{\prime}}{f}\right)^{2} \approx \lambda e^{-y / n}-\frac{(n+1)^{2}}{4 n^{2}} \quad y \gg 0 .
$$

Thus, for $\lambda$ sufficiently large there will be two turning points at $y=-\infty$ and $y=y_{0}$, where

$$
y_{0} \approx n \log \left(4 n \lambda /(n+1)^{2}\right)
$$

Hence, the WKB approximation for this curve gives

$$
(m+1 / 2) \pi=\int_{-\infty}^{y_{0}} d y \sqrt{\frac{\lambda}{f} e^{y}-\frac{1}{2} \frac{f^{\prime \prime}}{f}+\frac{1}{4}\left(\frac{f^{\prime}}{f}\right)^{2}}, \quad m \geq 0 .
$$

To leading order in $M$ we may approximate the WKB integral as

$$
\int_{-\infty}^{\infty} d y \sqrt{\lambda} e^{y / 2} f^{-1 / 2}=\int_{1}^{\infty} d x \frac{\sqrt{\lambda}}{\sqrt{x^{2+1 / n}-x}}=\frac{M}{T} \frac{\Gamma\left(\frac{1}{2+2 n}\right)}{4 \pi^{1 / 2} \Gamma\left(\frac{2+n}{2+2 n}\right)} .
$$

Let us now consider the next order term in the $1 / M$ expansion of ( (12.1 $\left.1 \overline{2}_{1}^{\prime}\right)$. There are two contributions to this constant piece. There is one contribution because (2.13) was integrated to $\infty$ instead of $y_{0}$. Hence we should subtract from (2. $\left.2_{2} \overline{3}_{1}\right)$ the term

$$
\int_{y_{0}}^{\infty} d y \sqrt{\frac{\lambda e^{y}}{f}}=(n+1)+\mathrm{O}(1 / \sqrt{\lambda})
$$

The other contribution comes from integrating the integrand in (ㄴ.2.12i $)$ near $y_{0}$. Subtracting off the leading order term, this contribution is given by

$$
\begin{gathered}
\int_{-\infty}^{y_{0}} d y \sqrt{\frac{\lambda}{f} e^{y}-\frac{1}{2} \frac{f^{\prime \prime}}{f}+\frac{1}{4}\left(\frac{f^{\prime}}{f}\right)^{2}}-\sqrt{\frac{\lambda e^{y}}{f}} \approx \\
\approx \int_{1}^{e^{y_{0}}} \frac{d x}{x^{1+1 /(2 n)}}\left(\sqrt{\lambda-\frac{(1+1 / n)^{2}}{4} x^{1 / n}}-\sqrt{\lambda}\right) \\
=\left(1-\frac{\pi}{2}\right)(n+1)+\mathrm{O}(1 / \sqrt{\lambda}) .
\end{gathered}
$$

We have used the fact that $f^{\prime \prime} / f$ and $f^{\prime} / f$ are almost constant near the turning point

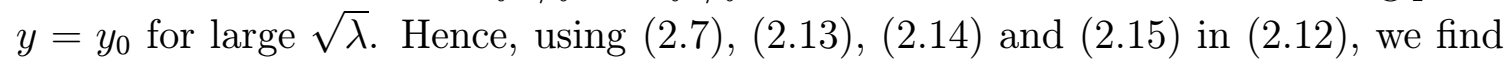
that

$$
M^{2}=16 \pi^{3}\left(\frac{\Gamma\left(\frac{2+n}{2+2 n}\right)}{\Gamma\left(\frac{1}{2+2 n}\right)}\right)^{2} T^{2} m(m+n)+\mathrm{O}\left(m^{0}\right), \quad m \geq 1 .
$$


In the $Q C D_{3}$ case, $n=1$ and hence the mass relation is

$$
\begin{aligned}
M^{2} & =8 \pi\left(\Gamma\left(\frac{3}{4}\right)\right)^{4} T^{2} m(m+1)+\mathrm{O}\left(m^{0}\right), \quad m \geq 1, \\
& \approx 5.74216(\pi T)^{2} m(m+1) .
\end{aligned}
$$

We have factored out a $\pi^{2}$ term in the second line of $\left(\overline{2} . \overline{1} \overline{7}_{1}\right)$ to match the units used in [9.9]1. Table compares the WKB expressions with the numerical results found in [9.] and we see that the agreement is very close. ${ }^{1}$

In the case of $Q C D_{4}$, we have $n=2$ and hence the WKB relation

$$
M=4 \pi^{3 / 2} \frac{\Gamma\left(\frac{2}{3}\right)}{\Gamma\left(\frac{1}{6}\right)} T \sqrt{m(m+2)}+\mathrm{O}\left(m^{-1}\right), \quad m \geq 1 .
$$

\begin{tabular}{|c|r|r|}
\hline$m$ & WKB & Numerical \\
\hline 1 & 11.4843 & 11.5877 \\
2 & 34.453 & 34.5270 \\
3 & 68.906 & 69.9750 \\
4 & 114.853 & 114.9104 \\
5 & 172.265 & 172.3312 \\
6 & 241.171 & 241.2366 \\
7 & 321.561 & 321.6265 \\
8 & 413.436 & 413.5009 \\
\hline
\end{tabular}

Table 1: Comparison of $0^{++}$ glueball masses squared in units of $\pi^{2} T^{2}$. The WKB approximation is very close to the numerical results in $\left[\begin{array}{l}\overline{9} \\ \overline{2}\end{array}\right]$, with a small difference approaching $0.064 \pi^{2} T^{2}$ for large $m$.

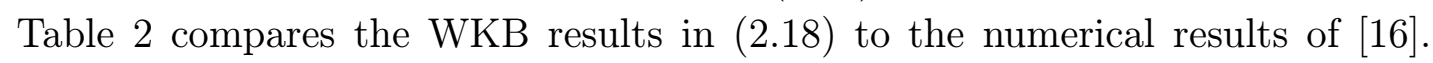
Again, we find that the WKB result quickly approaches the numerical eigenvalues.

We conclude this section by examining the behavior of the mass spectrum in the limit $n \rightarrow \infty$. Taking this limit we approach $p=5$, corresponding to six dimensional euclidean QCD with a compactified direction. In the large $n$ limit, the mass equation in ( $\left.2 . \overline{1} \cdot \overline{1} \overline{6}^{\prime}\right)$ reduces to

$$
M^{2}=4 \pi^{4} T^{2}\left(C+\frac{m}{n}\right), \quad m \geq 1,
$$

where $C$ is a constant to be determined. Thus, it appears that for nonzero $C$ there is a finite gap in the spectrum, but above this gap the spectrum is continuous. We can see this more clearly by taking $n \rightarrow \infty$ limit in $\left(\underline{2} . \overline{\sigma_{1}^{\prime}}\right)$. In this case $\left(\underline{2} . \overline{6}_{1}^{\prime}\right)$ reduces to Legendre's

\begin{tabular}{|c|c|c|}
\hline$m$ & WKB & Numerical \\
\hline 1 & 9.39 & 9.85 \\
2 & 15.3 & 15.6 \\
3 & 21.0 & 21.2 \\
4 & 26.5 & 26.7 \\
5 & 32.1 & 32.2 \\
6 & 37.6 & 37.7 \\
\hline
\end{tabular}

Table 2: Comparison of $0^{++}$ glueball masses for $Q C D_{4}$ in units of $T$. The WKB approximation should approach the numerical result as a function of $1 / m$. equation and so the solutions that are regular at $x=1$ are $P_{\ell}(2 x-1)$ where $\lambda=-\ell(\ell+1)$. If $\lambda \leq 1 / 4$ then $P_{\ell}(2 x-1)$ is not normalizable at infinity. If $\lambda>1 / 4$, then $P_{\ell}(2 x-1)$ is plane wave normalizable. Therefore, we find that the constant in ( $\left(2.1 \overline{1}_{1}^{\prime}\right)$ is $C=\pi^{-2}$ and thus there is a gap. This unusual behavior for the six dimensional theory is probably related to its nonlocal nature [1]

\footnotetext{
${ }^{1}$ In [פ్̣̂] a WKB expression was given with a numerical factor of 6 .
} 


\section{Glueball masses for generalized supergravity backgrounds}

In the previous section we have seen that the analytic WKB expressions give accurate results for the eigenvalues of the dilaton wave equation. This strengthens are confidence in the procedure, and encourages us to use it in more general situations.

In this section we discuss the WKB approximation for $0^{++}$glueballs in more general supergravity backgrounds. We will argue that that there is universality in the spectra which only depends on the dimensionality of the $A d S$ space at infinity and the singularity structure at the horizon. Using results derived here we can find WKB approximations for $0^{-+}$glueballs in $Q C D_{4}$ and $0^{--}$glueballs in $Q C D_{3}$. We can also explain the stability of the $0^{++}$spectrum for models coming from rotating branes [1] $13 \overline{-1}$, and the change in the spectrum for the $0^{-+}$glueballs in these same models.

The only assumptions that we make are that there are angular independent solutions to the dilaton wave equation in $\left(\underline{2} . \overline{4}_{1}^{1}\right)$ and that for large $U$ the metric approaches an $A d S$ solution. In this case, we can reduce (2.4i $)$ to

$$
\partial_{U}\left(f(U) \partial_{U} \phi\right)+M^{2} h(U)=0
$$

where

$$
f(U)=\sqrt{g} e^{-2 \phi} g^{U U}, \quad h(U)=\sqrt{g} e^{-2 \phi} g^{x x} .
$$

Let us suppose that there is a $U_{0}$ such that near $U=U_{0}$,

$$
f(U) \sim\left(U-U_{0}\right)^{s}, \quad h(U) \sim\left(U-U_{0}\right)^{q} .
$$

The assumption that asymptotically the solution is $A d S$ implies that $f(U) \sim$ $U^{8-p}$ and $h(U) \sim U$ if $U \gg U_{0}$.

Let us define $e^{y}=U-U_{0}, \tilde{f}(y)=f\left(e^{y}+U_{0}\right) e^{s y}$ and $\widetilde{h}(y)=h\left(e^{y}+U_{0}\right) e^{q y}$. If we let $\phi=\frac{e^{(1-s) y / 2}}{\sqrt{\widetilde{f}}} \psi$, then $\left(\underline{1} \overline{3} . \overline{1_{1}^{\prime}}\right)$ becomes

$$
\partial_{y}^{2} \psi+V(y) \psi=0
$$

where

$$
V(y)=M^{2} e^{(q+2-s) y} \frac{\widetilde{h}(y)}{\widetilde{f}(y)}+\frac{1}{4}\left(\frac{\partial_{y}\left(e^{(s-1) y} \tilde{f}(y)\right)}{e^{(s-1) y} \tilde{f}(y)}\right)^{2}-\frac{1}{2} \frac{\partial_{y}^{2}\left(e^{(s-1) y} \tilde{f}(y)\right)}{e^{(s-1) y} \tilde{f}(y)} .
$$

For large negative and positive $y$ we have

$$
\begin{array}{ll}
V(y) \approx C_{1} M^{2} e^{(q+2-s) y}-\frac{1}{4}(s-1)^{2} & y \ll 0, \\
V(y) \approx C_{2} M^{2} e^{(p-5) y}-\frac{1}{4}(7-p)^{2} & y \gg 0,
\end{array}
$$


where $C_{1}$ and $C_{2}$ are unimportant constants for this discussion. Hence, we find two turning points which for large enough $M$ can be approximated as

$$
\begin{aligned}
& y_{1} \approx-\frac{1}{q+s-2} \log \left(\frac{4 C_{1} M^{2}}{(s-1)^{2}}\right), \\
& y_{2} \approx \frac{1}{5-p} \log \left(\frac{4 C_{2} M^{2}}{(7-p)^{2}}\right) .
\end{aligned}
$$

The WKB approximation is then

$$
\left(m+\frac{1}{2}\right) \pi=\int_{y_{1}}^{y_{2}} d y \sqrt{V(y)} .
$$

The leading order contribution to the integral in (

$$
M \xi=M \int_{-\infty}^{+\infty} d y e^{y} \sqrt{\frac{e^{q y} \widetilde{h}(y)}{e^{s y} \widetilde{f}(y)}}=M \int_{U_{0}}^{\infty} d U \sqrt{\frac{g^{x x}}{g^{U U}}}
$$

where we have used (13.2). It is clear that $\left(\overline{3}_{3} \cdot \overline{9}_{1}^{\prime}\right)$ sets the inverse mass scale for the glueballs. We can compare this to the scale coming from the heavy quark potentials. In this latter case, the string tension along the brane, as a function of the energy scale $U$ is

$$
\sigma(U)=\frac{1}{2 \pi} g_{x x}(U)
$$

and at large quark separation the string tension approaches $\frac{1}{2 \pi} g_{x x}\left(U_{0}\right)$. Hence we

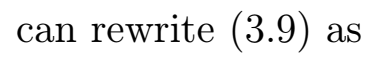

$$
M \xi=M \int_{U_{0}}^{\infty} d U \sqrt{g_{U U}}(2 \pi \sigma(U))^{-1 / 2}
$$

In other words, the inverse mass scale is a one-loop integral of the square root of the inverse tension integrated over all energy scales with a measure $\sqrt{g_{U U}}$.

Let us now consider the next to leading order corrections. The computations are similar to those in $\left(\overline{2} . \overline{1} \overline{4}_{1}^{\prime}\right)$ and $\left(\underline{2} . \overline{1} \overline{5}_{1}^{\prime}\right)$. Using $\left(\underline{3}_{3} \cdot \overline{6}_{1}^{\prime}\right)$, the correction coming from the turning point at $y=y_{2}$ is

$$
\begin{aligned}
- & \sqrt{M^{2} C_{2}} \int_{y_{2}}^{\infty} d y e^{(p-5) y / 2}+ \\
& +\int_{-\infty}^{y_{2}} d y\left(\sqrt{M^{2} C_{2} e^{(p-5) y}-\frac{1}{4}(7-p)^{2}}-\sqrt{M^{2} C_{2}} e^{(p-5) y / 2}\right)=-\left(\frac{7-p}{5-p}\right) \frac{\pi}{2}
\end{aligned}
$$

while the correction coming from the turning point at $y=y_{1}$ is

$$
\begin{aligned}
- & \sqrt{M^{2} C_{1}} \int_{-\infty}^{y_{2}} d y e^{(q+2-s) y / 2}+ \\
& +\int_{y_{2}}^{+\infty} d y\left(\sqrt{M^{2} C_{1} e^{(q+2-s) y}-\frac{1}{4}(s-1)^{2}}-\sqrt{M^{2} C_{2}} e^{(q+2-s) y / 2}\right)=-\frac{|s-1|}{q+2-s} \frac{\pi}{2} .
\end{aligned}
$$


Putting everything together, we find that the WKB masses are

$$
M^{2}=\frac{\pi^{2}}{\xi^{2}} m\left(m+\frac{2}{5-p}+\frac{|s-1|}{q+2-s}\right)+\mathrm{O}\left(m^{0}\right), \quad m \geq 1 .
$$

By assuming that the supergravity solution is asymptotically $A d S$, we have chosen a particular singularity structure for the point at spatial infinity. The basic arguments used here are still applicable even if the solution is not $A d S$, so long as the singularity structure at infinity is known.

We now consider some of the examples discussed in the recent literature. For

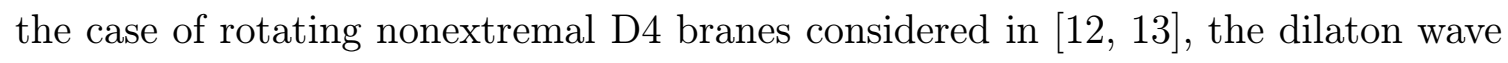
equation reduces to

$$
\partial_{u}\left[u\left(u^{6}-(4 g N)^{2} a^{4} u^{2}-u_{T}^{6}\right) \partial_{u} \phi\right]+4 g N M^{2} u^{3} \phi=0,
$$

where $a$ parameterizes the angular momentum, and we have replaced $U$ with $u^{2}=U$, to match the form of the equation in [1; 3 . If $a=0$ this reduces to the nonrotating D4 brane equation in ( $\left(2 . \overline{L_{1}}\right)$. The horizon occurs at $u=u_{0}$ with

$$
u_{0}^{6}-a^{4} u_{0}^{2}-u_{T}^{6}=0 .
$$

Hence, it is clear that (3.15i all values of $a$. Therefore, we find that the WKB expression for the masses is

$$
M^{2}=m(m+2) \frac{\pi^{2}}{4 g N}\left[\int_{u_{0}}^{\infty} d u \frac{u}{\sqrt{u^{6}-(4 g N)^{2} a^{4} u^{2}-u_{T}^{6}}}\right]^{-2}, \quad m \geq 1 .
$$

Since the singularity structure does not change when $a$ is varied, we see that the WKB mass ratios do not change either. Thus, we see the reason for the stability of the glueball masses observed in [i] $\mathbf{i}_{\mathbf{1}}$. This might also explain why supergravity glueball results are reasonably close to lattice results.

We can compute the integral in (15.17i) exactly in the two limits $a=0, \infty$. The result for $a=0$ is in (12.18). For large $a$ we find

$$
M^{2}=\frac{8}{\pi}\left(\Gamma\left(\frac{3}{4}\right)\right)^{4} a^{2} m(m+2), \quad m \geq 1 .
$$

The next examples are the $0^{-+}$glueball masses for the rotating nonextremal D4 branes. In this case, the equation of motion for one of the angular components of the R-R 1-form field is [i]

$$
\partial_{u}\left[u^{3}\left(u^{4}-(4 g N)^{2} a^{4}\right) \partial_{u} \chi\right]+4 g N M^{2} \frac{u^{5}\left(u^{4}-(4 g N)^{2} a^{4}\right)}{u^{6}-(4 g N)^{2} a^{4} u^{2}-u_{T}^{6}} \chi=0 .
$$

This equation also has the same form as ( $\left.\underline{b}_{-} . \overline{1}_{1}^{\prime}\right)$, but with $s=0$ and $q=-1$ for

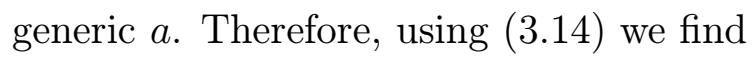

$$
M^{2}=m(m+3) \frac{\pi^{2}}{4 g N}\left[\int_{u_{0}}^{\infty} d u \frac{u}{\sqrt{u^{6}-(4 g N)^{2} a^{4} u^{2}-u_{T}^{6}}}\right]^{-2}, \quad m \geq 1,
$$


for the $0^{-+}$masses. In the $a=0$ limit this reduces to

$$
M=4 \pi^{3 / 2} \frac{\Gamma\left(\frac{2}{3}\right)}{\Gamma\left(\frac{1}{6}\right)} T \sqrt{m(m+3)}, \quad m \geq 1 .
$$

Table ${ }_{-1}^{13}$ ishows a comparison of the WKB masses to the numerical results in $[1 \overline{6} \overline{0}]$. The WKB result for the mass ratio between the lowest level $0^{-+}$and $0^{++}$states is $M_{-+} / M_{++}=2 / \sqrt{3} \approx 1.155$ which is reasonably close to the numerical result. In fact the difference between the WKB and numerical results is smaller

\begin{tabular}{|c|c|c|}
\hline$m$ & WKB & Numerical \\
\hline 1 & 10.8 & 11.8 \\
2 & 17.1 & 17.8 \\
3 & 23.0 & 23.5 \\
4 & 28.7 & 29.1 \\
5 & 34.3 & 34.6 \\
6 & 39.8 & 40.1 \\
\hline
\end{tabular}

Table 3: Comparison of $0^{-+}$ glueball masses for $Q C D_{4}$ in units of $T$. than present day lattice errors.

However, in the limit that $a \rightarrow \infty$, the WKB structure will change for the $0^{-+}$states. This is because the singularity structure of $\left({ }^{-} \overline{1}_{-1} \overline{9}_{1}^{\prime}\right)$ changes. In fact, in the large $a$ limit we end up with the same equation as the dilaton. Hence we find the same WKB masses. The only difference is that we have to discard the lowest eigenvalue [1] 13 . Hence the WKB mass ratio in the large $a$ limit is $\frac{M_{-+}}{M_{++}}=\sqrt{8 / 3} \approx$ 1.63. This is again close to the numerical result of 1.59 and it is also close to the lattice result of $1.61 \pm .19$.

Our final example is the WKB spectrum for the $\mathrm{O}^{--}$glueballs in $Q C D_{3}$. After a rescaling of the NSNS 2 form field, the relevant component satisfies the wave equation [9]

$$
\partial_{U}\left[U^{5}\left(U^{4}-U_{T}^{4}\right) \partial_{U} \chi\right]+g N M^{2} U^{5} \chi=0 .
$$

This does not have quite the same form as $\left({ }_{2} . \overline{1} \overline{1}_{1}\right)$ because of the extra $U^{4}$ term, but the WKB analysis is almost identical and results in the spectrum

$$
M=\sqrt{8 \pi}\left(\Gamma\left(\frac{3}{4}\right)\right)^{2} T \sqrt{m(m+3)}, \quad m \geq 1 .
$$

Table 'A' is a comparison of the WKB and numerical

\begin{tabular}{|c|r|r|}
\hline$m$ & WKB & Numerical \\
\hline 1 & 4.79 & 5.11 \\
2 & 7.58 & 7.82 \\
3 & 10.17 & 10.36 \\
4 & 12.68 & 12.84 \\
5 & 15.16 & 15.29 \\
6 & 17.61 & 17.73 \\
7 & 20.05 & 20.15 \\
8 & 22.48 & 22.57 \\
\hline
\end{tabular}

Table 4: Comparison of $0^{--}$ glueball masses in units of $\pi T$. results $[1 \overline{1} \overline{0}]$. Again, we find close agreement. Finally, the WKB mass ratio for the lightest states with $0^{--}$and $0^{++}$quantum numbers is $\frac{M_{--}}{M_{++}}=\sqrt{2}$.

\section{The type 0 nonsupersymmetric model.}

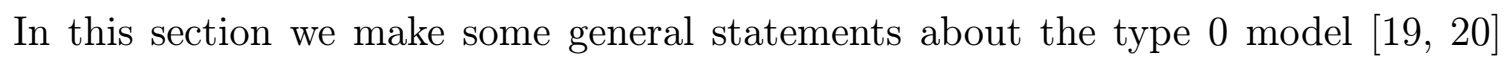
recently discussed in [1] 1 . We find asymptotic solutions for the lowest order supergravity approximation. We find a running of the effective coupling, but no confining behavior and no normalizable glueball solutions. 
The type 0 model has a closed string tachyon, no fermions and a doubled set of R-R fields. In particular, there is no longer a self dual constraint on the 5 -form field strength. Since the number of R-R fields is doubled, so are the number of D brane types [2]1]. Hence one can have D3 branes that are electric instead of dyonic. If we have $N$ parallel electric D3 branes, then the low energy effective action on the branes is thought to be $S U(N)$ QCD with adjoint scalar fields, but no fermions. Hence, there is no supersymmetry and the coupling will run. There is no open string tachyon [2]1], so there is no tachyon in this QCD model.

The authors in [1] 10 argued that the closed string tachyon can get an expectation value, and that its mass squared gets a positive shift from the background 5 form flux. The background tachyon field acts as a source for the dilaton, so the dilaton is no longer constant. One then makes the following ansatz for the metric [i]

$$
d s^{2}=e^{\frac{1}{2} \phi}\left(e^{\frac{1}{2} \xi-5 \eta} d \rho^{2}+e^{-\frac{1}{2} \xi} d x_{\|}^{2}+e^{\frac{1}{2} \xi-\eta} d \Omega_{5}^{2}\right),
$$

where $\phi, \xi$ and $\eta$ are functions of $\rho$ only. The equations of motion then reduce to a Toda like system with an action [1] $\left.\mathbf{1}_{-1}\right]^{2}$

$$
\begin{aligned}
S & =\int d \rho\left[\frac{1}{2} \dot{\phi}^{2}+\frac{1}{2} \dot{\xi}^{2}+\frac{1}{4} \dot{T}^{2}-5 \dot{\eta}^{2}-V(\phi, \xi, \eta, T)\right], \\
V(\phi, \xi, \eta, T) & =\frac{1}{2} T^{2} e^{\frac{1}{2} \phi+\frac{1}{2} \xi-5 \eta}+20 e^{-4 \eta}-Q^{2} f^{-1}(T) e^{-2 \xi},
\end{aligned}
$$

and a constraint

$$
\frac{1}{2} \dot{\phi}^{2}+\frac{1}{2} \dot{\xi}^{2}+\frac{1}{4} \dot{T}^{2}-5 \dot{\eta}^{2}+V(\phi, \xi, \eta)=0 .
$$

$Q$ is the total D3 brane charge which is proportional to $N, T$ is the tachyon field and $f(T)$ is a function given by 10 华

$$
f(T)=1+T+\frac{1}{2} T^{2}+\mathrm{O}\left(T^{3}\right) .
$$

For large $Q$, the tachyon expectation value is determined by setting $f^{\prime}(T)=0$. As a first approximation, we may assume that the tachyon is constant as a function of $\rho$.

If $T=0$ then the solution reduces to the $\mathcal{N}=4$ solution. When $T$ is nonzero, then all fields are coupled and there is no known analytic solution. However, we can attempt to find approximate solutions that are valid in the $U V$ and $I R$ regions. In the $U V$, we expect the dilaton field to be relatively constant, at least compared with $\xi$ and $\eta$. Assuming that $\phi$ is constant and thus ignoring its kinetic term, the equations for $\xi$ and $\eta$ can be solved exactly, at least in the near horizon limit. In this case we find

$$
e^{\xi}=C_{1} \rho, \quad e^{\eta}=C_{2} \rho^{1 / 2},
$$

\footnotetext{
${ }^{2}$ We are using units where $\alpha^{\prime}=1$.
} 


$$
\begin{aligned}
\frac{1}{4} T^{2} e^{\frac{1}{2} \phi} \frac{C_{1}^{1 / 2}}{C_{2}^{5}}+\frac{2 Q^{2}}{C_{1}^{2} f(T)}-1 & =0, \\
\frac{5}{2} T^{2} e^{\frac{1}{2} \phi} \frac{C_{1}^{1 / 2}}{C_{2}^{5}}+\frac{80}{C_{2}^{4}}-5 & =0 .
\end{aligned}
$$

One can easily check that this satisfies the constraint equation in ( $\left(\bar{A} . \overline{Z_{1}^{\prime}}\right)$. If we plug this back into the metric, we find that the solution is still $A d S_{5} \times S_{5}$, but the curvatures of the two spaces no longer match, $S_{5}$ now has smaller curvature then $A d S_{5}$. In this case the Ricci scalar for the total space is proportional to

$$
R \sim T^{2} e^{\frac{1}{2} \phi}
$$

Using the $\xi$ and $\eta$ solutions as inputs, we can go back and find an approximate solution for $\phi$ in terms of $\rho$. Using the ansatz $e^{\frac{1}{2} \phi}=C_{0}(\log \rho)^{\alpha}$, and plugging this into the equation of motion for $\phi$

$$
\ddot{\phi}+\frac{1}{4} T^{2} e^{\frac{1}{2} \phi+\frac{1}{2} \xi-5 \eta}=0,
$$

we find that the ansatz is a leading order solution if $\alpha=-1$ and $C_{0}=-8 C_{2}^{5} /\left(T^{2} \sqrt{C_{1}}\right)$. Setting $\rho=u^{-4}$, and using the lowest order solutions for $C_{1}$ and $C_{2}$ from ( $\left(\begin{array}{l}\overline{4} \\ -\end{array} \overline{5}_{1}^{1}\right)$, we learn that the leading order behavior for the coupling is

$$
e^{-\phi}=\frac{1}{g_{Y M}^{2}}=\frac{Q T^{4}(\log u)^{2}}{4096 \sqrt{2 f(T)}} .
$$

Thus we find a running coupling, but instead of a linear log dependence, the coupling runs with a log squared! One can easily check that to leading order in $1 / \log u$, the constraint equation is still satisfied. We can also estimate the range of validity for this solution. Computing the leading order corrections to $C_{1}$ and $C_{2}$, one finds that

$$
C_{1}=\frac{2 Q}{\sqrt{2 f(T)}}\left(1+\frac{1}{4 \log u}\right), \quad C_{2}=2\left(1+\frac{1}{4 \log u}\right) .
$$

We can also compare the terms in the potential that depend on the tachyon. Since

$$
\frac{1}{4} T^{2} e^{\frac{1}{2} \phi+\frac{1}{2} \xi-5 \eta} \sim \frac{u^{8}}{2 \log u}, \quad Q^{2} f^{-1}(T) e^{-2 \xi} \sim \frac{u^{8}}{2},
$$

our solution with a constant $T$ with $f^{\prime}(T)=0$ is valid so long as $\log u \gg 1$.

The metric in the large $u$ limit is

$$
d s^{2}=\frac{32}{T^{2} \log u}\left(\frac{d u^{2}}{u^{2}}+\frac{\sqrt{2 f(T)}}{2 Q}\left(1+\frac{1}{\log u}\right) r^{2} d x_{\|}^{2}+\left(1+\frac{1}{\log u}\right) d \Omega_{5}^{2}\right) .
$$

Hence we can trust the supergravity solution only if $T \ll 1$, since $\log u \gg 1$. However, it is clear that $T \sim 1$ if $f^{\prime}(T)=0$, hence one should not expect the 
supergravity result to be particularly trustworthy. Indeed, we have found that while the supergravity computation results in a coupling running to zero, it runs with the wrong power of $\log u$.

Nevertheless, the effective coupling between a heavy quark and its antiquark does appear with the expected log dependence. Using the Wilson line computation of $[2 \sqrt[2]{2}, 23]$, one finds that quark potential is given by

$$
V \approx-\frac{256 \pi^{3}}{\Gamma\left(\frac{1}{4}\right)^{4} T^{2} L \log \left(L_{0} / L\right)}, \quad L \ll L_{0},
$$

where we have plugged $R^{2}=\frac{32}{T^{2} \log u}$ into the expressions derived in $\left[2 \overline{2} 2,12 \overline{3}, 1 . L_{0}\right.$ is some length of order the string scale. Recall that the $\mathcal{N}=4$ potential comes with a coefficient $\sqrt{g N}$. It is this coefficient, and not the 't Hooft-Polyakov tension $g N$, that plays the role of $1 / \alpha^{\prime}$ for the supergravity models. For a string theory with extrinsic curvature, the string tension has a $(\log u)^{-1}$ dependence $\left[2 \overline{2} \overline{4}, \overline{2}, \overline{2} \overline{5_{1}}\right]$. Hence our result is in line with rigid string results, so long as one remembers that the tension is $\sqrt{g N}$.

Even though we cannot really trust the supergravity solution for large $u$, we might be able to trust it for small $u$. However, here we will see that the situation is even worse. In particular, we will find that the dilaton wave equation in the lowest order supergravity background has no normalizable glueball solutions. Moreover, the heavy quark potential is not confining.

In order to study the $I R$ behavior, let us follow the suggestion of [1] ind search for solutions for the toda system assuming that the second term in $V$ is small compared to the other terms. This corresponds to a small curvature for the $S_{5}$. Dropping this second term in $V$ and assuming a constant $T$, one can now find an exact solution to the equations of motion that satisfies the constraint. The solution is

$$
e^{\phi}=C_{0}^{2} \rho^{5 / 9}, \quad e^{\eta}=C_{2} \rho^{5 / 9}, \quad e^{\xi}=\frac{3 Q}{\sqrt{2 f(T)}} \rho,
$$

with the relation

$$
20(2 f(T))^{1 / 4} C_{2}^{5}-9 T^{2} \sqrt{3 Q} C_{0}=0 .
$$

Comparing all terms in $V$, one has $e^{\frac{1}{2} \phi+\frac{1}{2} \xi-5 \eta} \sim \rho^{-2}, e^{-2 \xi} \sim \rho^{-2}$, but $e^{-4 \eta} \sim \rho^{-20 / 9}$. Hence this solution is valid for large $\rho$. From ( and after substituting $\rho=1 / u^{4}$ the metric is

$$
d s^{2}=\frac{20}{9 T^{2}}\left(16 \frac{d u^{2}}{u^{2}}+\frac{C_{2}^{5} \sqrt{2 f(T)}}{3 Q} u^{8 / 9} d x_{\|}^{2}+C_{2}^{5} u^{-8 / 9} d \Omega_{5}^{2}\right) .
$$

$C_{2}$ remains as a leftover integration constant.

In the small $u$ limit, the $T$ dependent terms in the potential are now comparable, so the tachyon expectation value is no longer at $f^{\prime}(T)=0$. Instead, plugging in the 


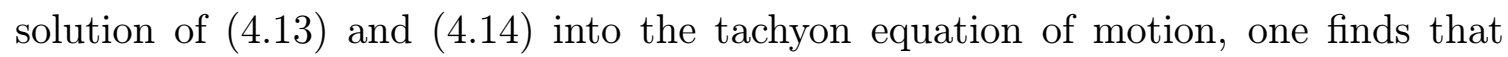
$\ddot{T}=0$ if

$$
10 f(T)+T f^{\prime}(T)=0 .
$$

Using the function in ( (1.4. for $T$. However, if we were to include the cubic term in $f(T)$ or include the quartic term $c_{1} T^{4}$ in the action, then a real solution would exist.

As in the $U V$, the curvature in the $I R$ is small if $T \ll 1$. However, we expect solutions to $\left(\overline{1} . \overline{1} \bar{b}^{\prime}\right)$ to be $T \sim 1$. The curvature is now at the string scale, so we cannot truely trust the supergravity solution in this limit either.

We can attempt to find glueball solutions. Using the asymptotic expressions in the dilaton equation of motion we find the equations

$$
\begin{aligned}
\partial_{u}\left[u^{5} \partial_{u} \phi\right]+\frac{2 Q M^{2}}{\sqrt{2 f(T)}} u \phi & =0, & & \text { »1, } \\
\partial_{u}\left[u^{5} \partial_{u} \phi\right]+\frac{48 Q M^{2}}{C_{2}^{5} \sqrt{2 f(T)}} u^{21 / 9} \phi & =0, & & u \ll 1 .
\end{aligned}
$$

For large $u$, we see that the dilaton equation of motion reduces to the $\mathcal{N}=4$ result. It appears that the only other singularity is at $u=0$. Using the arguments of the previous section, one learns from ('A. $\left.\bar{A}_{-} \overline{1}_{i}\right)$ that this singularity has $s-q=5-21 / 9>2$, hence no glueball solutions are possible.

We can also easily see that the potential between the heavy quarks does not confine for these solutions. If we define a new variable $v$ such that

$$
v=\frac{1}{9}\left(\frac{\sqrt{2 f(T)}}{3 Q}\right)^{1 / 2} u^{4 / 9}
$$

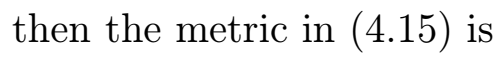

$$
d s^{2}=\frac{180}{T^{2}}\left(\frac{d v^{2}}{v^{2}}+v^{2} d x_{\|}^{2}+\frac{\left(C_{2} / 3\right)^{10} \sqrt{2 f(T)}}{Q^{2} v^{2}} d \Omega_{5}^{2}\right)
$$

From this metric, we see that $R^{2}=\frac{180}{T^{2}}$, and so the heavy quark potential is

$$
V \approx-\frac{1440 \pi^{3}}{\Gamma\left(\frac{1}{4}\right)^{4} T^{2} L}, \quad L \gg L_{0} .
$$

Hence, the supergravity result implies that the effective heavy quark coupling increases when going from the $U V$ to the $I R$, but the quark potential does not develop a linear term and remains coulombic.

We could certainly generate a linear quark potential by going to finite temperature since the supergravity background would now have a horizon at finite $u$. The 
down side of this scenario is that the the theory will essentially be reduced to QCD in three dimensions.

In the end one probably has to consider the full $\sigma$-model in order to get a linear quark potential and normalizable glueball solutions. At the very least, one could try including the $\alpha^{\prime 3} R^{4}$ terms in the action to see if this would qualitatively change the behavior in the infrared. Perhaps one could then combine the $U V$ results found here with $I R$ results derived from the $\sigma$-model to say something concrete about the WKB glueball masses.

\section{Conclusions}

We have seen from the WKB mass expressions that there is some degree of universality for glueball mass ratios between different supergravity models. In the examples where the ratios change, the behavior can be attributed to a change of the singularity structure at the horizon.

The finite temperature models do not exhibit a running of the coupling in the $U V$. However, we have shown that the type 0 model has the desired behavior. Unfortunately, it does not appear to be confining in the $I R$. Hopefully confinement will appear in the solution for the full $\sigma$-model. Or perhaps a model can be found that combines the desired features of the nonsupersymmetric models discussed here.

\section{Acknowledgments}

I would like to thank O. Bergman, E. Gimon, P. Horava and D. Minic for discussions. This work was supported in part by funds provided by the DOE under grant number DE-FG03-92-ER40701.

\section{References}

[1] J. Maldacena, The large $N$ limit of superconformal field theories and supergravity,

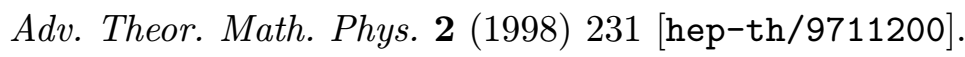

[2] S. Gubser, I. Klebanov and A. Polyakov, P'Phys. [hep-th/9802 $10 \bar{g}^{\prime}$.

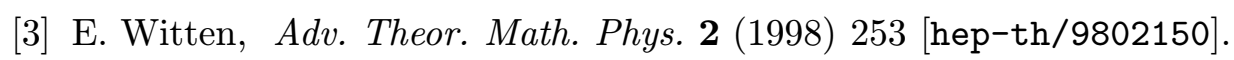

[4] E. Witten, Anti-de Sitter space, thermal phase transition, and confinement in gauge

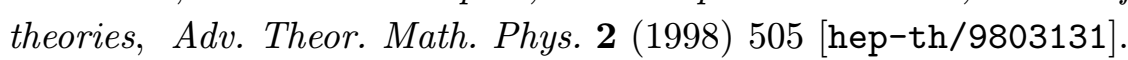

[5] S.-J. Rey, S. Theisen and J. Yee, Wilson-Polyakov loop at finite temperature in large

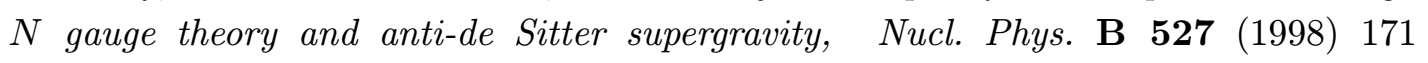
[hep-th/9803135i. 
[6] A. Brandhuber, N. Itzhaki, J. Sonnenschein and S. Yankielowicz, Wilson loops, confinement, and phase transitions in large $N$ gauge theories from supergravity, $\bar{U}_{-}^{-} \bar{H} \overline{i g} \bar{h}_{1}$

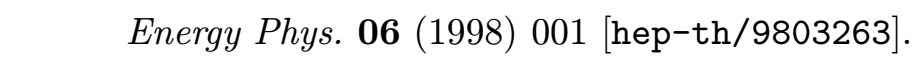

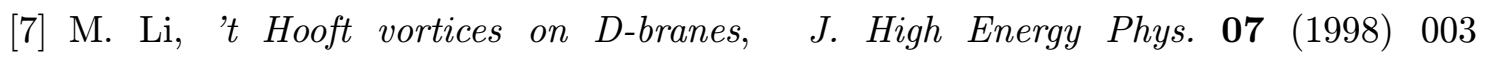
[hep-th/9803252i]; 't Hooft vortices and phases of large $N$ gauge theory, - - E E

[8] D. Gross and H. Ooguri, Phys. Rev. D 58 (1) 998$)$ 106002 [hep-th/9805129].

[9] C. Csaki, H. Ooguri, Y. Oz and J. Terning, Glueball mass spectrum from supergravity, hep-th/9806021:

[10] R. de Mello Koch, A. Jevicki, M. Mihailescu and J.P. Nunes, 'Phys. Rev. D 58 i

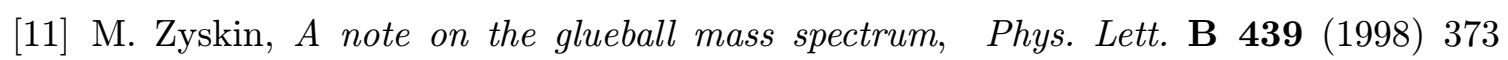
[hep-th/9806128il.

[12] J. Russo, New compactifications of supergravities and large $N Q C D$, hep-th/9808117i.

[13] C. Csaki, Y. Oz, J. Russo and J. Terning, Large N QCD from rotating branes, 'Phys.'

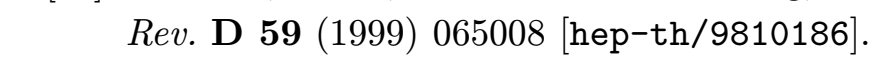

[14] I. Klebanov and A. Tseytlin, D-branes and dual gauge theories in Type 0 strings, hep-th/9811035.

[15] S. Gubser, I. Klebanov and A. Peet, Phys. Rev. D. 54 (1996] 391

[16] A. Hashimoto and Y. Oz, Aspects of QCD dynamics from string theory, hep-th/9809106.

[17] O. Aharony, M. Berkooz, D. Kutasov and N. Seiberg, Linear dilatons, NS5-branes and holography, 过. High Energy Phys. 10

[18] A. Peet and J. Polchinski, UV/IR relations in AdS dynamics, hep-th/9809022i,

[19] L. Dixon and J. Harvey, iNucl. Phys. B $\mathbf{2} 74(1986) 93$

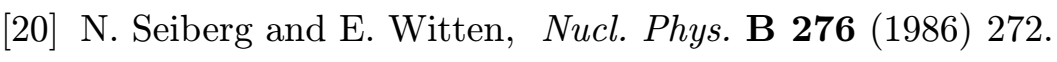

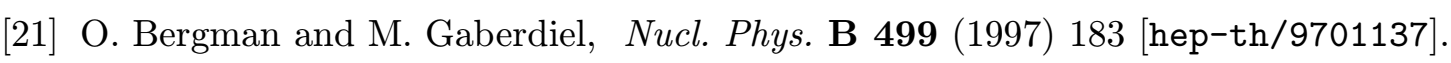

[22] S.-J. Rey and J. Yee, Macroscopic strings as heavy quarks in large $N$ gauge theory and anti-de Sitter supergravity, hep-th/9803001:

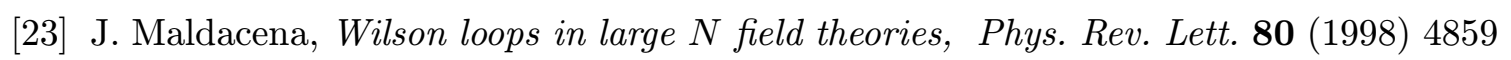
[hep-th/9803002in.

[24] A. Polyakov, iNucl. Phys. B 268 (1986)

[25] P. Horava, On QCD string theory and AdS dynamics, hep-th/9811028. 